\title{
On the Ideal Ratio Mask as the Goal of Computational Auditory Scene Analysis
}

\author{
Christopher Hummersone, Toby Stokes and Tim Brookes
}

\begin{abstract}
The ideal binary mask (IBM) is widely considered to be the benchmark for time-frequency-based sound source separation techniques such as computational auditory scene analysis (CASA). However it is well known that binary masking introduces objectionable distortion, especially musical noise. This can make binary masking unsuitable for sound source separation applications where the output is auditioned. It has been suggested that soft masking reduces musical noise and leads to a higher quality output. A previously defined soft mask, the ideal ratio mask (IRM), is found to have similar properties to the IBM, may correspond more closely to auditory processes, and offers additional computational advantages. Consequently the IRM is proposed as the goal of CASA. To further support this position, a number of studies are reviewed that show soft masks to provide superior performance to the IBM in applications such as automatic speech recognition and speech intelligibility. A brief empirical study provides additional evidence demonstrating the objective and perceptual superiority of the IRM over the IBM.
\end{abstract}

\section{Introduction}

A natural environment usually consists of a number of sound sources. Some may convey information that is important to the listener (a person speaking for example), whilst others may be less important (a distant vehicle for example). If the important information is considered to be a target signal and all other sound is considered to be noise/interference, then this situation may be modelled as

Christopher Hummersone

University of Surrey, Guildford, UK, e-mail: c.hummersonedsurrey.ac.uk

Toby Stokes

University of Surrey, Guildford, UK, e-mail: t.stokes@surrey.ac.uk

Tim Brookes

University of Surrey, Guildford, UK, e-mail: t.brookes@surrey.ac.uk 


$$
z(n)=x(n) * h(n)+d_{\mathrm{st}}(n)+d_{\mathrm{nst}}(n)
$$

where $z$ is the mixture at sample index $n, x$ is the target signal, $h$ is the acoustic/channel impulse response, and $d_{\mathrm{st}}$ and $d_{\mathrm{nst}}$ are stationary and non-stationary noise/interference respectively [33]. In certain situations these unhelpful interfering sound source(s) may prevent the listener from receiving all of the information from the important target sound source. A machine listener, such as an automatic speech recognition (ASR) system, may also be impeded by the presence of interfering sounds. But in a natural environment acoustic interference is often inescapable. Hence reducing the level of acoustic interference may be useful in a number of applications, including: ASR, speaker identification, human-computer interaction, audio information retrieval, and hearing prostheses. This broad range of applications has meant that blind source separation (BSS) is an important area of research in signal processing and related fields.

Through the course of research, four main approaches to BSS have emerged: independent component analysis (ICA), spatial filtering, non-negative matrix factorisation (NMF), and computational auditory scene analysis (CASA). ICA seeks to separate components based on statistical independence. The technique aims to find the inverse mixing matrix that provides the most independent separated source signals [23, 7, 34]. Spatial filtering uses microphone array signal processing to enhance sound arriving from a particular direction. NMF [24] aims to factorise a timefrequency (T-F) representation of a mixture in to two matrices: bases and coding. The bases matrix is formed from a set of unique spectral structures; each basis does not represent a source in the mixture but rather each sound that is part of the mixture. For example, the signal from a piano would be divided into each individually occurring note or speech into individual formants. The coding matrix determines the temporal activation of the bases. CASA aims to mimic human auditory scene analysis (ASA) [9], which is the process by which a human makes sense of an auditory scene, a key part of which is the separation of mixtures of sounds. Humans demonstrate a remarkable ability to extract a target sound from a mixture, providing important motivation for research into CASA. It is for this reason that the current chapter chooses to focus on CASA.

A typical CASA system broadly consists of two stages [50]. Firstly an analysis of the audio in the T-F domain is used to decide whether a particular T-F unit should be designated as target or interference. Secondly, this information is used to mask the $\mathrm{T}-\mathrm{F}$ representation in order to reduce or eliminate the interference. In his seminal treatise on the topic, Wang [48] proposed the ideal binary mask (IBM) as the goal of CASA. The IBM is set to one when the target energy exceeds the interference energy and zero otherwise. Binary masking has also been coupled with other aforementioned BSS techniques including ICA (e.g. [37]) and NMF (e.g. [18]). The proposal of the IBM as the goal of CASA has been supported by a number of studies that have shown the IBM to be advantageous for machine and human listening tasks, including speech intelligibility (e.g. [11, 40, 27]), and ASR (e.g. [42, 13, 19]). Furthermore, it was shown that under certain constraints the IBM is the optimal binary mask in terms of signal-to-noise ratio (SNR) [28]. 
However, it is well known that the binary mask separation method introduces audible distortions, especially so-called musical noise. The distortion is caused by repeated narrow-frequency-band switching. As will be shown in this chapter, the perceived audio quality of binary-masked audio is poor. This has the potential to limit the applications of binary mask-based techniques such as CASA to domains where the output is not auditioned. This constitutes a significant limitation.

In order to address this limitation and the shortcomings of the IBM, this chapter will propose the ideal ratio mask (IRM) [42] as the goal of CASA. Under certain circumstances and/or for particular applications, the value of the IBM may be great, and this chapter is not intended to refute that. Instead, the chapter will argue that the IRM may be preferable to the IBM as the goal of CASA for a number of theoretical and practical reasons, and across a majority of applications. The chapter will start with a review of the musical noise problem is Sect. 2. A number of advantageous features of the IRM will then be reviewed in Sect. 3 , leading to the proposal of the IRM as the goal of CASA. The IBM and IRM will then be compared in Sect. 4 using existing studies and a brief empirical study utilising both purely objective and perceptually-informed objective BSS metrics.

\section{The Problem with the Ideal Binary Mask}

Although binary masking has proved to be an effective BSS method, the prevalence of artefacts such as musical noise appears to have a deleterious effect on the audio quality of the separated output. Whilst this might not be problematic for applications where the output is not auditioned (such as ASR or databasing tasks) for other tasks (such as speech enhancement or auditory scene reconstruction) the poor audio quality is likely to prevent adoption of binary mask-based techniques such as CASA.

Few studies have compared the audio quality achieved by binary masking to that achieved by other BSS methods, and hence it is difficult to draw meaningful conclusions on the degree to which binary masking is deleterious for audio quality. Some data can be found in Table 1 [33]. The paper compares four BSS algorithms against IBM-based separation. The first model, M1, combines a noise tracker based on voice activity detection (VAD) with a minimum mean-square-error (MMSE) spectral amplitude estimator (STSA) [15]. The second model, M2, combines a VAD-based noise tracker with a log-spectral amplitude estimator (LSA) [16]. M1 and M2 use the "decision-directed" method [15] to estimate the a priori SNR by weighting the estimated spectral amplitude and noise variance of the previous frame, and the $a$ posteriori SNR in the current frame. The third model, M3, uses a magnitude-DFT MMSE estimator under the assumption that the required coefficients have a generalised Gamma distribution (GGD). The fourth model, proposed in the paper, combines a noise estimator designed for highly non-stationary noise (NSNE) [39] with a maximum likelihood (ML) speech estimator to produce a DFT-based soft mask. The comparison utilises the perceptual evaluation of audio source separation (PEASS) 
Table 1 Performance data [33] comparing the unprocessed noisy mixture with the output of a number of BSS algorithms (M1-M3), a proposed BSS algorithm [33], and the IBM. The comparisons are in terms of OPS, and SNR (in dB).

\begin{tabular}{llrrrrrrr}
\hline & & \multicolumn{7}{c}{ Target-to-Interference Ratio (dB) } \\
\cline { 4 - 8 } Noise Estimation + Method & Metric & -6 & -3 & 0 & 3 & 6 & 9 & Average \\
\hline -+ Noisy speech [12] & OPS & 9.4 & 8.6 & 25.9 & 8.6 & 9.2 & 18.2 & 13.3 \\
& SNR & -8.2 & -3.0 & -0.6 & 2.6 & -2.8 & 6.3 & -1.0 \\
M1: VAD + LSA [15] & OPS & 19.7 & 15.7 & 30.6 & 28.9 & 34.4 & 40.9 & 28.3 \\
& SNR & -6.7 & -0.8 & 1.4 & 4.3 & -1.7 & 5.4 & 0.3 \\
M2: VAD + STSA [16] & OPS & 20.4 & 16.2 & 31.9 & 29.4 & 34.4 & 37.5 & 28.3 \\
& SNR & -6.6 & -0.8 & 1.4 & 4.2 & -1.7 & 5.2 & 0.3 \\
M3: MMSE [20] + GGD [17] & OPS & 21.8 & 19.3 & 26.3 & 27.9 & 31.7 & 28.1 & 25.8 \\
\multirow{2}{*}{ NSNE + ML [33] } & SNR & 0.6 & 0.9 & 1.2 & 1.1 & 0.9 & 1.2 & 1.0 \\
& OPS & 27.0 & 21.8 & 45.4 & 34.0 & 33.4 & 50.3 & 35.3 \\
& SNR & 2.1 & 3.1 & 4.5 & 4.8 & 4.7 & 6.2 & 4.2 \\
\hline Ideal + IBM & OPS & 16.6 & 12.4 & 13.3 & 14.4 & 14.0 & 13.9 & 14.1 \\
& SNR & 4.4 & 5.5 & 5.1 & 5.1 & 3.6 & 4.3 & 4.7 \\
\hline
\end{tabular}

toolbox [14]. The results indicate that in terms of "overall perceptual score" (OPS) (a metric intended to indicate the "global quality" of the separated output), the IBM performs poorly compared to the other methods; the difference is as much as 20 points on the 100-point scale.

A similar trend can be observed in Table 2] [4], which compares: S1, a generalised expectation-maximisation framework for handling prior information [35]; S2, a $k$ subspace-based tensor factorisation method [31]; the IBM obtained via the shorttime Fourier transform (STFT); and the IBM obtained via the gammatone filterbank (GTFB). The table shows some differences of a similar magnitude to Table 1, depending on the T-F decomposition and mixture. Note that the SNR and signal-todistortion ratio (SDR) data presented in the tables show that poor OPS performance is not solely attributable to poor separation performance.

Other studies have shown that the IBM is not optimal in terms of audio quality. One study [10] found that although the IBM improves speech intelligibility in noisy conditions and causes the noise to be less annoying, the separated speech is unnat-

Table 2 Data from SiSEC2011 [4], for tasks $\mathrm{T} 2$ or $\mathrm{T} 3$ and instantaneously mixed dataset D1, showing the average OPS and SDR (in $\mathrm{dB}$ ) of a number of BSS techniques, including the IBM.

\begin{tabular}{llrrrr}
\hline \multirow{2}{*}{ System } & Metric & $\begin{array}{r}2 \text { mic } \\
\text { speech }\end{array}$ & $\begin{array}{r}2 \text { mic } \\
\text { 3 music }\end{array}$ & $\begin{array}{r}2 \text { mic } \\
\text { speech }\end{array}$ & $\begin{array}{r}3 \text { mic } \\
\text { speech }\end{array}$ \\
\hline S1 [35] & OPS & 43.9 & 52.3 & 42.4 & - \\
& SDR & 13.4 & 16.6 & 8.9 & - \\
S2 [31] & OPS & 43.2 & 40.0 & 29.8 & 39.7 \\
& SDR & 7.9 & 6.9 & 3.0 & 11.7 \\
\hline IBM (STFT) & OPS & 38.9 & 33.3 & 27.1 & - \\
& SDR & 10.8 & 10.4 & 9.1 & - \\
IBM (GTFB) & OPS & 24.0 & 30.4 & 22.0 & - \\
& SDR & 8.5 & 9.0 & 7.5 & - \\
\hline
\end{tabular}


Table 3 A comparison using the PEASS metrics of the IBM with three mask postprocessing and/or alternative mask estimation algorithms [43].

\begin{tabular}{|c|c|c|c|c|}
\hline \multicolumn{5}{|c|}{ Method APS IPS TPS OPS } \\
\hline IBM & 12 & 76 & 51 & 18 \\
\hline DBM & 29 & 62 & 67 & 36 \\
\hline NBM & 48 & 66 & 67 & 49 \\
\hline CBM & 53 & 61 & 66 & 49 \\
\hline
\end{tabular}

ural and consequently listeners do not find it preferable to the unseparated mixture. It is noted that by softening the mask the distortions are reduced and the noise is increased (lowering intelligibility), but that the result is preferred by listeners to the IBM and un-processed outputs. It should be noted that this study was conducted on normal hearing listeners. There is some evidence to suggest that hearing-impaired listeners are less sensitive to musical noise [1]. Therefore it may not be advantageous to lower the SNR by softening the mask for applications targeting hearing-impaired listeners.

A number of other studies have also shown that musical noise arising from binary masking can be reduced by soft masking, but that this comes at a cost in terms of SNR (e.g. [25, 2, 5]).

Such is the disturbance caused by musical noise that some studies have attempted to improve the perceptual quality of binary-masked audio (e.g. [2, 3, 29]). In one study, summarised in Table 3 , mask post-processing, and alternative mask estimation algorithms, were compared to the IBM in an attempt to improve the OPS of the separated output [43]. Specifically, the study compared: the IBM; a noisy binary mask (NBM) that had triangular probability density function (TPDF) noise added to the binary values; a dithered binary mask in which the SNR had TPDF dither added prior to mask calculation; and a cepstrally-smoothed binary mask [29] that, after optimisation, effectively added 0.1 to all zero-valued mask units. Similarly to previous studies noted above, the study concluded that the OPS could be improved, but at the cost of some interference suppression. Although the DBM demonstrated some quality improvement, methods that demonstrated the greatest improvement in OPS allowed the mask values to deviate from zero and one.

These results suggest that a well-defined soft mask may achieve a better audio quality than a binary mask. This has also been suggested by other authors [29, 49]. However, it seems that the choice of soft mask should be made carefully such that it does not introduce an SNR penalty. Several authors (e.g. [6, 5]) have suggested the use of sigmoid functions in order to generate soft masks. One such approach [5] showed that a soft mask defined in this way offers a slight signal-to-interference ratio (SIR) advantage over a binary mask. However, it remains unclear how such sigmoidal masks perform using more common metrics such as SNR. One mask that has received attention in recent years is the IRM [42]. As will be shown in the next section, the IRM has a number of properties that make it a good alternative to the IBM as the goal of CASA. 


\section{The Ideal Ratio Mask as the Goal of Computational Auditory Scene Analysis}

CASA aims to model the human process of ASA [48]. Bregman [9] states that the goal of ASA is "the recovery of separate descriptions of each separate thing in the environment". However, this goal is too vague to be transferred directly to CASA. In his important treatise on the goal of CASA, Wang [48] suggests three options, before suggesting that the IBM should be the goal. The first option is to separate out all sound sources in a given mixture. However, this goal is far beyond the capabilities of the human listener who may only be able to separate a handful of concurrent sound sources. The second option is to enhance ASR. Whilst appealing, since this is one of the primary applications of CASA, it is not the only application. Thus in order to retain maximum usefulness across applications, the goal should not be tied to a specific application. The final option is to enhance human listening. However, not all applications involve human listeners (ASR, for example), and thus this goal would also only apply to a subset of applications. Measuring the responses of human listeners may also introduce prohibitive requirements of time, resources, and/or expertise that might hinder progress in the field.

Consequently, Wang [48] suggests that the IBM should be the goal of CASA, for a number of reasons discussed in this section. In contrast, this section proposes the IRM as the goal of CASA. The thesis is based on three strands of argumentation: that the IRM matches or exceeds all of the desirable properties laid out by Wang (Sect. 3.1); that the IRM provides a closer match to psychophysical and perceptual mechanisms than the IBM (Sect. 3.2); and that the IRM provides a number of computational advantages (Sect. 3.3.

\subsection{Properties of the IBM and IRM}

Firstly, for the sake of clarity, the IBM $\mathbf{m}_{B}$ and IRM $\mathbf{m}_{R}$ are defined in the following way:

$$
\begin{aligned}
& \mathbf{m}_{B}(c, m)=\left\{\begin{array}{ll}
1 & \frac{\mathbf{u}_{t}(c, m)}{\mathbf{u}_{i}(c, m)}>1 \\
0 & \text { otherwise }
\end{array},\right. \\
& \mathbf{m}_{R}(c, m)=\frac{\mathbf{u}_{t}(c, m)}{\mathbf{u}_{t}(c, m)+\mathbf{u}_{i}(c, m)},
\end{aligned}
$$

where $\mathbf{u}_{\{t, i\}}$ is the power of the target and interfering source(s) ( $t$ and $i$ respectively) in time frame $m$ and frequency bin/channel $c$.

In his paper proposing the IBM as the goal of CASA, Wang [48] specified four desirable properties of the IBM. These are: 
1. "flexibility"-for a given mixture, the mask will differ according to which sources are designated target and interference;

2. "well-definedness" - the ideal mask retains its definition independently of how many sources are present;

3. ceiling performance- the IBM is the optimal binary mask; and

4. psychoacoustical correspondence-the IBM broadly agrees with auditory masking and ASA [9] theories.

Given the similarity of the definitions of the IBM and IRM shown in (2) and (3), it can be seen that the IRM shares all of these properties. Firstly, the IRM is identically flexible: any source can be designated as the target, and the sum of remaining sources is typically designated as the interference. Secondly, the IRM is also welldefined, since the interference component may constitute any number of sources. Thirdly, the IRM is the optimal ratio mask and is closely related to the ideal Wiener filter, which is the optimal linear filter with respect to MMSE [51, 28]. Lastly, the IRM broadly agrees with psychoacoustic principles. This last point might seem surprising: it might appear counterintuitive that the IBM and IRM can both honour psychoacoustic principles. However, they can and, although they are both approximations, the next section will show how the IRM is perhaps a better approximation of auditory masking and ASA principles than the IBM.

\subsection{Psychophysical and Perceptual Bases of the IRM}

It is argued by Wang [48] that the IBM corresponds closely to auditory masking and ASA theories. However, this section argues that the IRM provides a closer match.

The concept of binary masking assumes that auditory masking is dichotomous: that a sound is either masked or it is not. To put it another way, it suggests that a sensory threshold exists. However, it has been known since the 1950s that this is an inadequate description when discussing sensory perception in any modality (see [44] for a review). Like any sensory threshold, auditory masking is only dichotomous in the sense that the experimenter asks the subject a yes/no question, e.g. "is the sound audible?". It seems reasonable that under identical circumstances a listener should always give the same answer. However, this is often not the case. The probability of a consistent answer depends on the relative level of the competing stimulus: the greater the difference, the greater the probability of a consistent answer. Furthermore, individual listeners may give different answers. In auditory masking experiments this probability of detection is often plotted as a function of signal magnitude in order to produce a psychometric function.

Auditory masking, like many aspects of sensory perception, has therefore been described using signal detection theory [38, 46, 45]. When applied to the auditory domain, the theory defines a decision variable, which often corresponds to physiological or psychological responses to a stimulus, such as the auditory nerve firing rate or sensory impression. Signal detection theory dictates that the average value of the decision variable is monotonically related to stimulus magnitude. However, 
signal detection theory also dictates that the value of the decision variable may fluctuate. There are two causes of fluctuation: external variations such as background noise level, or internal variations such as plausible differences in neural responses or other psychological factors [32].

It can be seen therefore that a ratio mask, where values vary in the range $[0,1]$, provides a closer match to signal detection theory than a binary mask. A value of 0 or 1 indicates certainty in the absence or presence of a signal, respectively. An intermediate value may be obtained when the signals are of similar magnitude. Although this may or may not agree with an experimentally-derived psychometric function, it at least provides a conceptual indication that masking is uncertain.

It should be noted, however, that the concept of a binary mask does agree to some extent with the ASA [9] theory. The theory draws on Gestalt principles of "exclusive allocation" (sometimes referred to as "disjoint allocation" or "belongingness") in visual perception, whereby a sensory element can not be used in the descriptions of more than one object at a time. However, whilst this principle generally holds true, there are a number of examples of violations of this principle in the auditory domain (see [8] for a review). Bregman [8] describes this as "duplex perception". Furthermore, the grouping of sensory elements may depend on perspective or attention. Unlike the ratio mask, a binary mask can not account for these observations because each T-F unit is always assigned to the source with the most energy.

In his paper, Wang [48] compares auditory objects to visual objects. Using this analogy, foreground visual objects are assigned a mask value of one, whereas occluded objects are assigned a value of zero. However, Bregman [8] argues that

... sound is transparent. A sound in the foreground does not 'occlude' a sound in the back-

ground in the same way as a visual object occludes our view of objects behind it.

In the visual domain, objects are occluded because light emitted or reflected from the object does not impinge on the retina. In the auditory domain, even if a sound source is visually occluded, its acoustic energy still usually impinges on the ear drums via numerous acoustic pathways. Thereafter sounds are occluded by physiological, psychophysical, or psychological mechanisms, rather than an absence of stimulating input to the auditory system. Furthermore, the human head seldom occludes sound; sound arriving at both ears usually contains information about all sound sources.

Given that auditory objects are transparent, it seems disadvantageous to assign portions of the sensory input to only one object when information about both objects is available. A visual analogy is given in Figure 1. The two images on the left show two objects that are overlapped such that there is now a small common area. Using a disjoint allocation principle — demonstrated in the middle of the figure- the common area must be assigned to one object. The scenario is analogous to binary masking. The occluding object is corrupt whereas the occluded object is incomplete. Using a duplex perception principle-demonstrated in the right of the figure-the common area may be assigned to both objects. The scenario is analogous to ratio masking. The resulting objects are now complete, irrespective of the chosen source, although each is corrupted to some extent by the other. The ratio value indicates the extent of the corruption and hence how meaningful the area is likely to be to the cur- 


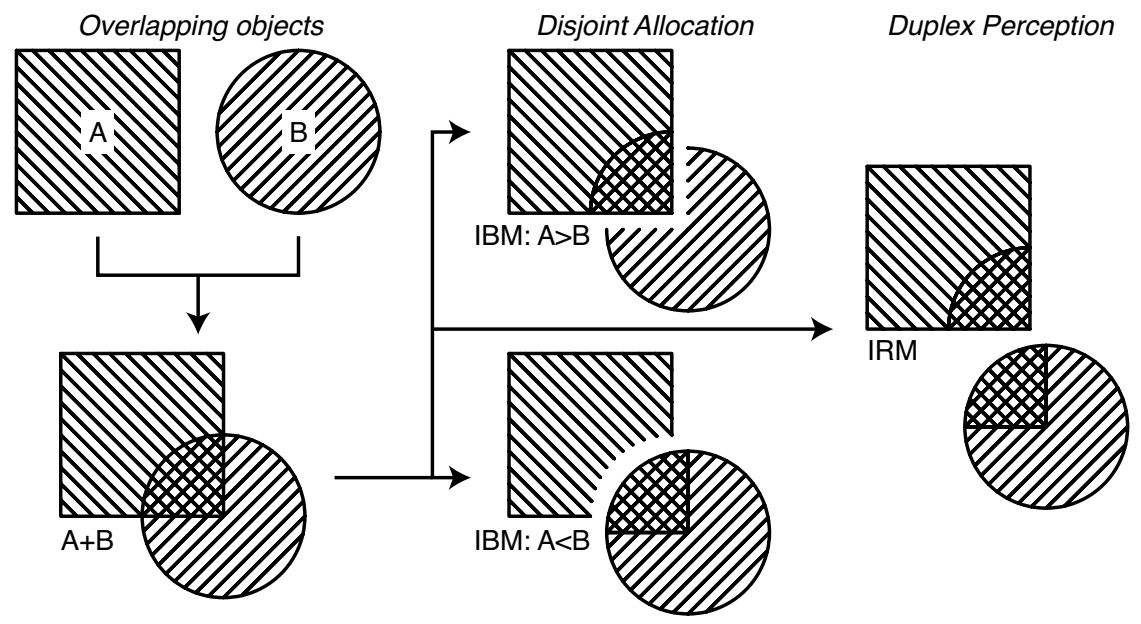

Fig. 1 Visual analogies of disjoint allocation and duplex perception when objects overlap (left): the disjoint allocation case (middle) is analogous to the binary mask; the duplex perception case (right) is analogous to the ratio mask

rent source. Note that this analogy applies not only to overlapping auditory objects, but also to the BSS problem since algorithms often try to estimate some parameter(s) of both the occluding and occluded signal in order to decide how to assign $\mathrm{T}-\mathrm{F}$ units ( $(2)$ and $(3)$ assume that some knowledge of both sources is available).

\subsection{Computational Bases of the IRM}

The IRM has a number of theoretical computational advantages, which are discussed in this section.

In their important paper on the relative merits of the IBM and IRM, Li \& Wang [28] note that:

... the IRM achieves higher SNR gains compared to the IBM. However, despite the fact that the IBM is binary and the IRM is not, the SNR gain of the IBM is surprisingly close to that of the IRM. This shows that the IBM is a very reasonable performance metric for sound separation. Indeed, there are reasons to prefer the IBM over the IRM as the computational goal of a separation system. The estimation of the IBM is considerably simpler than that of the IRM: the former requires only binary decisions, while the latter requires estimating the energy ratio of the two signals. Binary estimation is facilitated by the existence of numerous classification and clustering methods.

The SNR achieved by the IRM is shown in the paper to be, on average, $0.1-0.8 \mathrm{~dB}$ higher than that achieved by the IBM, depending on the T-F decomposition and constituent signals. Of course, the importance of this gain depends on the overall SNR of the BSS algorithm. 
Li \& Wang argue above that binary masking facilitates classification and clustering methodologies. Whilst the IRM may preclude these possibilities, it does facilitate alternative probabilistic frameworks; the ratio value may be considered to indicate the probability that a given T-F region is reliable [6] or belongs to a particular source. Additionally, the binary mask can be considered as a special case and subset of the ratio mask. Indeed the equivalent binary mask may always be derived from the ratio mask by rounding or quantising ratio values; the IRM can not be derived from the IBM. This is important from an application point of view, and suggests that algorithms should always try to estimate the IRM; the algorithm may subsequently choose (or be told) to quantise the mask if the application, for whatever reason, deems it appropriate.

Lastly, Li \& Wang suggest that estimating ratio values may be more difficult than making binary decisions. Whilst this may or may not be the case, it should first be considered that the original definitions of the IBM (2) and IRM (3) contain identical quantities: estimates of the target and total interfering signal energy. Hence, in principle the task does not differ in its complexity, even if practical applications do not intend to estimate these values directly. Furthermore, simplicity is a relativistic concept; undoubtedly as knowledge in this field advances estimates of the IRM will become more accurate. The next section will consider the concept of "difficulty" in more detail.

\section{Comparisons of the Ideal Binary and Ratio Masks}

Thus far this chapter has outlined theoretical and practical reasons to prefer the IRM over the IBM. The IRM has been shown to offer a small SNR gain over the IBM [28]. It has also been suggested that soft masks may provide superior audio quality to binary masks. A number of other studies have provided reasons to prefer soft masks to binary masks.

In the original paper proposing the IRM [42], summarised in Table 4 it was found that, for a small vocabulary digit recognition task, the IBM coupled with a missing-data ASR system marginally out-performed the IRM coupled with a conventional ASR system, by an accuracy of the order of $1 \%$ across all SNRs. However,

Table 4 A comparison of the IBM coupled with a missingdata ASR system and the IRM coupled with a conventional ASR system for two different vocabulary sizes [42].

\begin{tabular}{rccccc}
\hline & \multicolumn{4}{c}{ Accuracy [\%] } \\
\cline { 2 - 5 } $\begin{array}{c}\text { Small } \\
\text { Vocabulary }\end{array}$ & \multicolumn{2}{c}{$\begin{array}{c}\text { Large } \\
\text { Vocabulary }\end{array}$} \\
\cline { 2 - 3 } \cline { 5 - 6 } SNR [dB] & IRM & IBM & & IRM & IBM \\
\hline-5 & 94.8 & 94.9 & 96.4 & 66.5 \\
0 & 95.7 & 96.0 & 97.0 & 71.2 \\
5 & 96.4 & 97.2 & 97.6 & 76.5 \\
10 & 97.7 & 98.1 & 97.7 & 80.1 \\
$\infty$ & 98.6 & 97.2 & 97.7 & 82.7 \\
\hline
\end{tabular}


Table 5 A comparison of fuzzy and binary masks coupled with missing-data ASR performing a digit recognition task under different noise conditions [6].

\begin{tabular}{|c|c|c|c|c|}
\hline \multirow[b]{3}{*}{ SNR $[\mathrm{dB}]$} & \multicolumn{4}{|c|}{ Digit Recognition Accuracy [\%] } \\
\hline & \multicolumn{2}{|c|}{ Factory Noise } & \multicolumn{2}{|c|}{$\begin{array}{c}\text { Lynx Helicopter } \\
\text { Noise }\end{array}$} \\
\hline & Fuzzy & Binary & Fuzzy & Binary \\
\hline 0 & 60 & 46 & 86 & 77 \\
\hline 5 & 81 & 73 & 95 & 92 \\
\hline 10 & 90 & 87 & 98 & 96 \\
\hline 15 & 95 & 95 & 99 & 98 \\
\hline 20 & 97 & 97 & 99 & 99 \\
\hline 200 & 99 & 99 & 99 & 99 \\
\hline
\end{tabular}

with a large vocabulary command and control task there was demonstrable improvement from the IRM of as much as $30 \%$ accuracy, with greatest improvement found in higher noise conditions. Similar findings, shown in Table 5, were made [6] by employing a soft "fuzzy" (though non-ideal) mask rather than a binary (non-ideal) mask. In this case, the fuzzy mask was produced by compressing the difference between the estimated local noise and signal $x$ using a sigmoidal function of the form

$$
f(x)=\frac{1}{1+e^{-\alpha(x-\beta)}}
$$

where $\alpha \in[0, \infty)$ and $\beta \in[0,1]$ are parameters controlling the sigmoid slope and centre respectively. The binary mask was derived in a similar way; the final values were rounded to 0 or 1 . As shown in the table, the fuzzy mask achieves an ASR accuracy gain of a more modest $14 \%$, again with greatest improvement found in higher noise conditions.

Gains have also been observed for human audition. A recent study [30] found that a soft mask based on the Wiener filter (IWF) significantly out-performed the IBM (with either a fixed or local threshold, IBM-F and IBM-L respectively) in terms of

Table 6 Speech intelligibility of speech separated using different T-F masks and under different interference conditions [30].

\begin{tabular}{|c|c|c|c|c|c|c|}
\hline \multirow{4}{*}{$\frac{\mathrm{SNR}[\mathrm{dB}]}{-35}$} & \multicolumn{6}{|c|}{ Correct [\%] } \\
\hline & \multicolumn{3}{|c|}{ Babble Interference } & \multicolumn{3}{|c|}{ Speech Interference } \\
\hline & \multicolumn{3}{|c|}{$\overline{\text { IWF IBM-L IBM-F }}$} & \multicolumn{3}{|c|}{ IWF IBM-L IBM-F } \\
\hline & 96 & 50 & - & 87 & 4 & - \\
\hline-30 & 100 & 29 & - & 92 & 10 & - \\
\hline-25 & 98 & 52 & - & 98 & 23 & - \\
\hline-20 & 96 & 62 & - & 96 & 71 & 13 \\
\hline-15 & 100 & 56 & - & 100 & 87 & 25 \\
\hline-10 & - & 62 & 2 & - & 85 & 69 \\
\hline-5 & - & 75 & 38 & - & 92 & 87 \\
\hline 0 & - & 90 & 75 & - & 100 & 100 \\
\hline 5 & - & 90 & 98 & - & - & - \\
\hline 10 & - & 87 & 96 & - & - & - \\
\hline
\end{tabular}


Table 7 Speech intelligibility of masked speech with speech-shaped-noise interference [22] for a variety of masking algorithms.

\begin{tabular}{rrrrr}
\hline & \multicolumn{4}{c}{ Intelligibility [\%] } \\
\cline { 2 - 5 } SNR [dB] & \multicolumn{4}{c}{ CG-MMSE BG-HU Noisy BG2-MMSE } \\
\hline-8 & 50.5 & 21.2 & 46.2 & 38.2 \\
-6 & 69.8 & 36.3 & 56.0 & 55.7 \\
-4 & 77.2 & 48.6 & 68.9 & 68.9 \\
-2 & 87.4 & 64.3 & 82.8 & 74.5 \\
0 & 89.5 & 79.1 & 91.1 & 85.8 \\
\hline
\end{tabular}

speech intelligibility and quality. The speech intelligibility data are summarised in Table 6. In this study the formulation of the IWF was identical to the IRM since the power-spectral density was not smoothed. The table shows that the intelligibility gain can be as much as $100 \%$ in high noise conditions. A similar finding, summarised in Table 7, was made in another recent study [22], although the data were yielded from non-ideal masks. In the paper, the authors created a number of mask-estimation algorithms based on estimating the MMSE of the spectral magnitude. Specifically, a continuous gain MMSE mask (CG-MMSE) was compared with with two binary gain (BG) estimators (BG-HU [21] and BG2-MMSE proposed in the work). The unprocessed noisy speech was also tested. The table shows that the CG-MMSE system achieved a more modest gain of up to approximately $30 \%$ in higher noise conditions. These differences in performance were attributed, in part, to the better preservation of the target envelope by the soft mask. It has been shown that the signal envelope is important for speech intelligibility [41].

These studies have offered compelling evidence that the IRM may provide a superior output to the IBM for a number of applications. It was also shown [30] that the Wiener filtering approach is less sensitive to errors in terms of speech intelligibility. However, it remains unclear how the IBM and IRM trade off in terms of the numerous sources of error and the magnitude of any separation performance gains, and whether a ratio mask retains its error robustness in terms of sound source separation metrics (i.e. whether the supposed difficulty of estimating the IRM [28] incurs a penalty). The rest of this section describes a new study that attempted to address these points. The study compared the separated audio output produced by binary and ratio masks using a number of objective and perceptually-informed objective metrics. As in the previously reported study [30] it was assumed that task difficulty could be modelled by introducing errors to the target and interfering signal energy. The errors inevitably led to an erroneous mask. Consequently two additive error components $\varepsilon_{t}$ and $\varepsilon_{i}$ were introduced, resulting in "estimated" binary and ratio masks ( $\hat{\mathbf{m}}_{B}$ and $\hat{\mathbf{m}}_{R}$ respectively).

The error components were calculated independently for the target and interfering signal using a previously defined method [30]. Specifically the error perturbed the spectral coefficient(s) in each $\mathrm{T}-\mathrm{F}$ unit prior to the calculation of spectral power used to formulate the masks. However, unlike the previous study [30], both the short-time Fourier transform (STFT) and the gammatone filterbank (GTFB) were utilised. Similarly to Li \& Wang's study [28] the GTFB was fourth-order and had 64 
channels with centre frequencies equally spaced on the ERB-rate scale between 50 and $8000 \mathrm{~Hz}$, although the time and phase responses were aligned using the method described by Patterson et. al. [36]. The STFT was 512-point but the frames were not overlapping (since Li \& Wang [28] point out that the IBM may only be optimal when frames do not overlap).

The spectral power for the target and interferer signals, $\hat{\mathbf{u}}_{t}$ and $\hat{\mathbf{u}}_{i}$ respectively, were calculated in the following way. For the GTFB

$$
\hat{\mathbf{u}}_{\{t, i\}}(c, m)=\sum_{n=m M_{G T}}^{(m+1) M_{G T}-1}\left[\mathbf{X}_{\{t, i\}}(c, n)+\theta \varepsilon_{\{t, i\}}(c, m)\right]^{2},
$$

where $\mathbf{X}$ is the output of the GTFB for the target or interfering signals, $M_{G T}$ is the frame length ( $10 \mathrm{~ms}$ in samples), and $n$ is the sample index. For the STFT

$$
\hat{\mathbf{u}}_{\{t, i\}}(c, m)=\left|\sum_{n=M_{F F T} m}^{(m+1) M_{F F T}-1} x_{\{t, i\}}(n) \mathrm{e}^{-\mathrm{j} 2 \pi \frac{c}{M_{F F T}} n}+\theta \varepsilon_{\{t, i\}}(c, m)\right|^{2},
$$

where $M_{F F T}$ is the FFT size (512). In both cases $\varepsilon$ is an error component and $\theta \in[0,1]$ is the error magnitude. For the GTFB the error was normally distributed noise with zero mean. For the STFT the error was complex noise where both real and imaginary parts were normally distributed with zero mean. In each case the error was scaled in each frequency channel/bin to have equal power to the unperturbed target and interferer signals. The perturbed powers were used to calculate "estimated" binary and ratio masks, $\hat{\mathbf{m}}_{B}$ and $\hat{\mathbf{m}}_{R}$ respectively, such that

$$
\hat{\mathbf{m}}_{B}(c, m)= \begin{cases}1 & \frac{\hat{\mathbf{u}}_{t}(c, m)}{\hat{\mathbf{u}}_{i}(c, m)}>1 \\ 0 & \text { otherwise }\end{cases}
$$

and

$$
\hat{\mathbf{m}}_{R}(c, m)=\frac{\hat{\mathbf{u}}_{t}(c, m)}{\hat{\mathbf{u}}_{t}(c, m)+\hat{\mathbf{u}}_{i}(c, m)} .
$$

With $\theta=0$, the signal power was unperturbed and the masks were ideal, with $\theta=1$ the error had equal magnitude to the unperturbed signals. The estimated masks were applied to the unperturbed mixture in order to calculate the performance metrics. Examples of the masks are shown in Figures 2 and 3

The stimuli were taken from the SiSEC2013 corpu\$ Instantaneous mixtures from the D1 and D2 data sets were used; each source was designated in turn as the target, with the sum of remaining sources designated as the interference (for the purposes of calculating $\hat{\mathbf{u}}_{i}$ and $\varepsilon_{i}$ ).

In total eight metrics were employed:

- SNR;

1 http://sisec.wiki.irisa.fr/tiki-index.php 


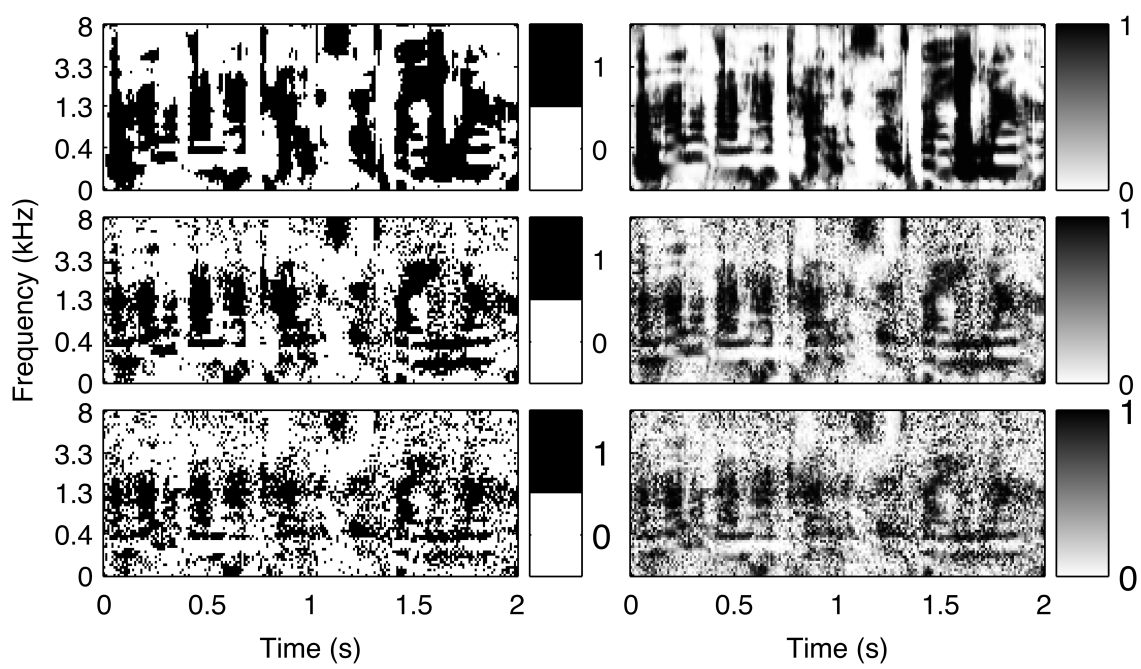

Fig. 2 Examples of the ideal and "estimated" masks using a gammatone filterbank: binary masks (left column) and ratio masks (right column); ideal masks $(\theta=0)$ (top row), $\theta=0.5$ (middle row), and $\theta=1$ (bottom row)
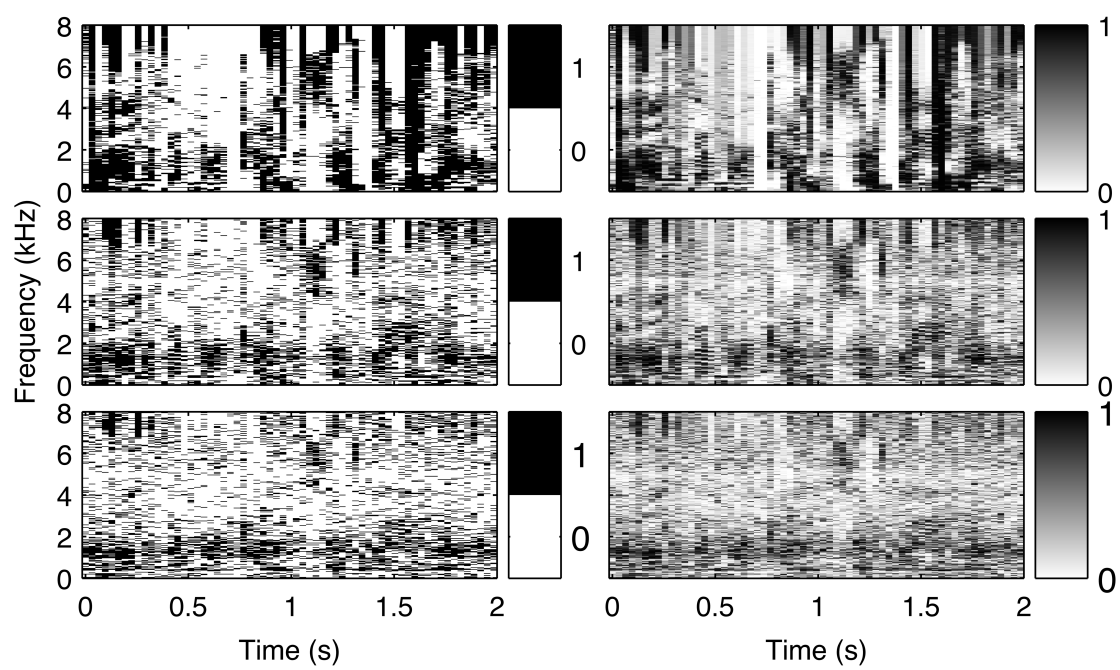

Fig. 3 Examples of the ideal and "estimated" masks using a short-time Fourier transform: binary masks (left column) and ratio masks (right column); ideal masks $(\theta=0)$ (top row), $\theta=0.5$ (middle row), and $\theta=1$ (bottom row)

- three metrics from the BSS_eval toolbox [47]: SDR, SIR, and signal-to-artefacts ratio (SAR); and 

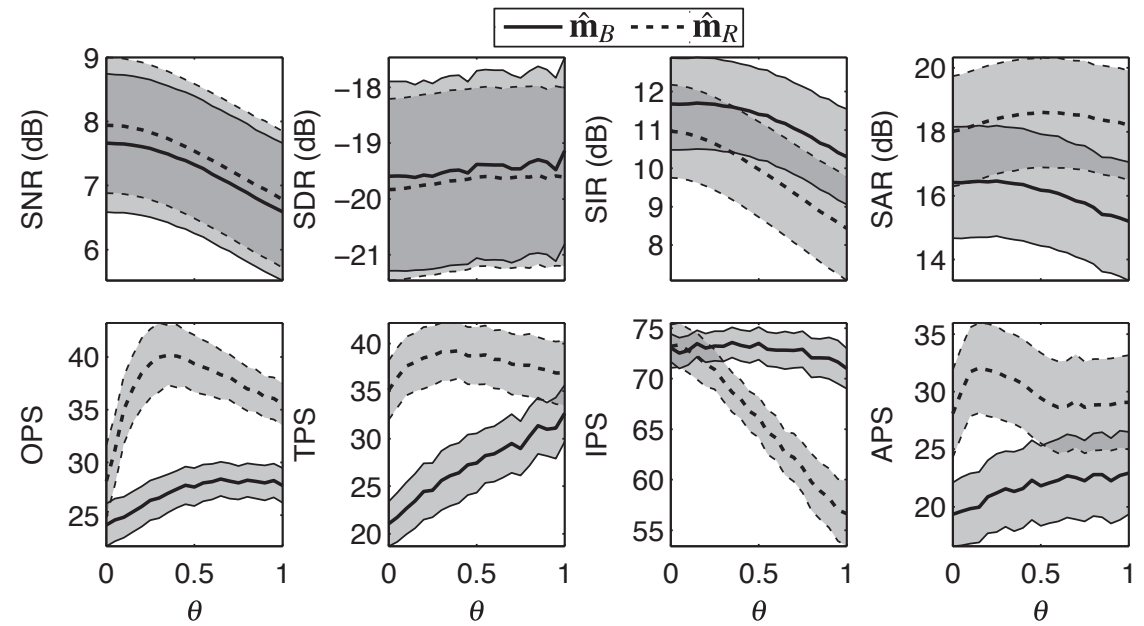

Fig. 4 Performance of binary and ratio masks under error conditions, using a gammatone filterbank, measured using a number of objective and perceptually-informed objective metrics; grey regions show $95 \%$ confidence intervals
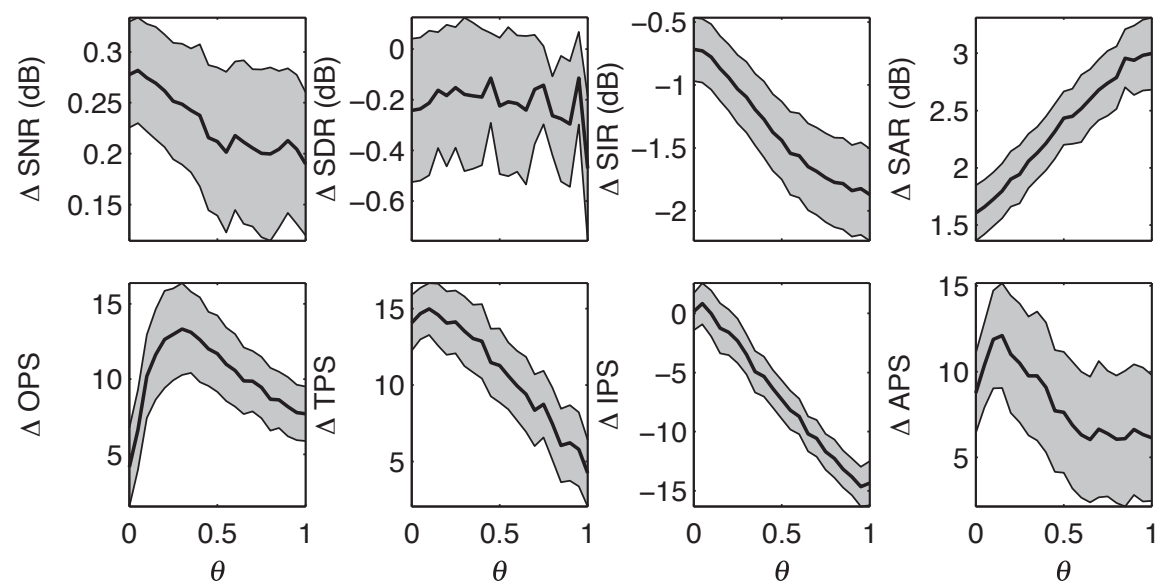

Fig. 5 Differences in mask performance for data shown in Fig. 4 (scores for the binary masks are subtracted from scores for the ratio masks); grey regions show $95 \%$ confidence intervals

- four metrics from the PEASS toolbox [14]: OPS, target-related perceptual score (TPS), interference-related perceptual score (IPS), and artefact-related perceptual score (APS).

The SNR and OPS metrics are designated here as "global" metrics, since they produce a single quantity derived from a number of sources of error, whereas the other metrics consider only a subset of error sources. 

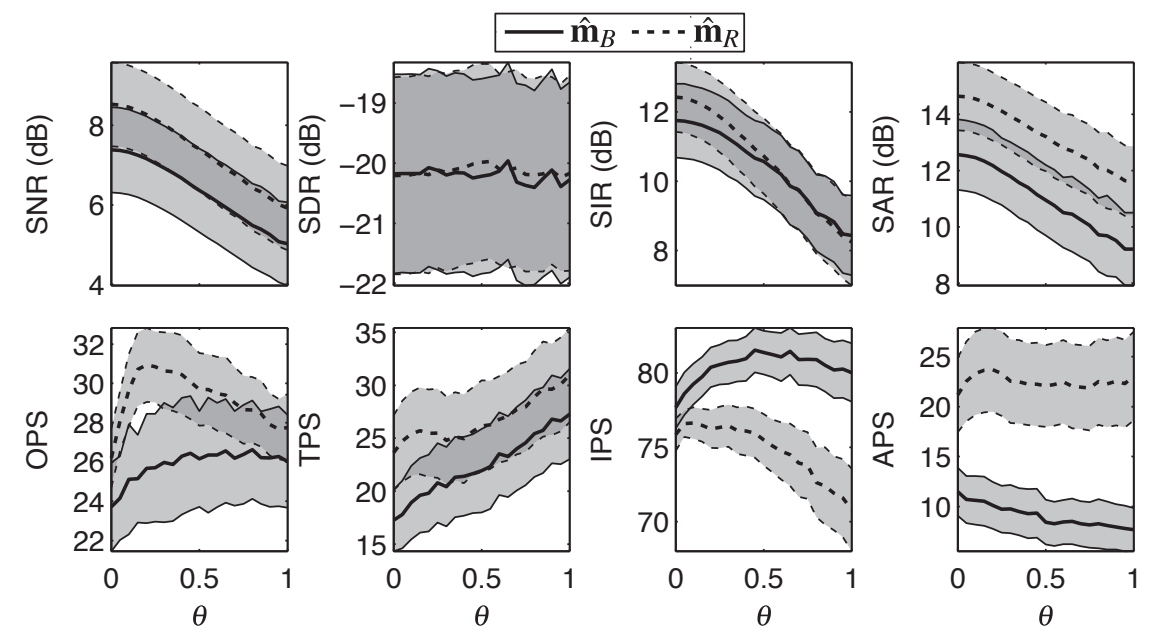

Fig. 6 Performance of binary and ratio masks under error conditions, using a short-time Fourier transform, measured using a number of objective and perceptually-informed objective metrics; grey regions show $95 \%$ confidence intervals
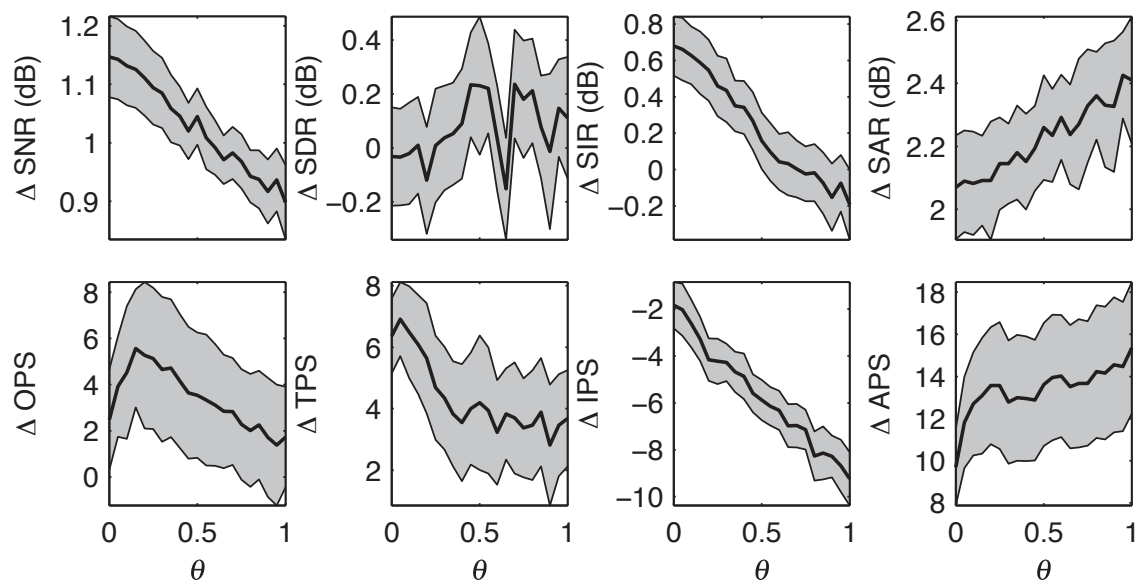

Fig. 7 Differences in mask performance for data shown in Fig. 6 (scores for the binary masks are subtracted from the scores for the ratio masks); grey regions show $95 \%$ confidence intervals

The results for the GTFB versus error magnitude $\theta$ are shown in Fig. 4 Differences, calculated as the scores for the ratio masks minus the scores for the binary masks, are given in Fig. 5. Note that in some cases the confidence intervals in Fig. 4 overlap but the corresponding difference scores in Fig. 5 are significant, i.e. the lower bound of the confidence interval is greater than zero. This is because calculating differences eliminates within-group variation, so that only between-group 
variation is exposed. The results show that the ratio masks retain a small but significant SNR advantage over the binary masks for all $\theta$. The ratio masks are also superior in terms of OPS, TPS, SAR, and APS. However, this it at the cost of interference suppression (SIR and IPS). The difference in SDR is negligible. The reduction in artefacts is particularly prominent, and seems to have resulted in a substantial improvement in OPS for some values of $\theta$. Informal listening suggested that the increase in OPS is attributable to the reduction in musical noise.

In terms of error resilience, the results show that no significant penalty is incurred when there are errors in estimating source power. The ratio masks retains their SNR, OPS, TPS, SAR, and APS advantage for all $\theta$.

Data for the STFT are given in Figs. 6 and 7 The trends shown in these data appear to mostly align with observations made of the GTFB. Note that the SNR gain is generally larger in this case, whereas the OPS gain is generally smaller. Interestingly, the ratio masks out-perform the binary masks in terms of SIR for most values of $\theta$, although this advantage is not reflected in the IPS.

These results suggest that the ratio masks out-perform the binary masks in terms of the "global" metrics (SNR and OPS). As previously found, the IRM's SNR gain over the IBM is small, and this advantage is retained when the masks deviate from ideal. The ratio masks appear to significantly reduce musical noise, out-performing the binary masks in terms of artefact reduction (SAR and APS). They also appear to generally out-perform the binary masks in terms of TPS. However, the cost here has been shown to be interference suppression. It remains unclear whether this will be important to any specific applications. Therefore the IRM seems likely to be a more appropriate goal for most applications. It should be noted that these differences may depend upon the T-F resolution, especially in terms of speech intelligibility [26, 30], such that smaller differences may be observed at higher resolutions.

It may be of interest to note that both experiments indicate a maximal OPS for $\theta>0$, i.e. for a non-ideal mask. It seems that the introduction of random errors into an ideal mask can reduce the severity of objectionable artefacts (as can be seen in the APS plots) due to a lessening of the regularity and, for a ratio mask, the severity of transitions. This phenomenon has been explored in a previous study [43].

\section{Conclusions}

This chapter has argued that the IRM is a more appropriate goal for CASA than the IBM. A number of reasons, summarised in the following sentences, were provided. Firstly, the IRM shares desirable properties with the IBM that make it an appropriate goal for CASA. Secondly, the IRM seems to correspond more closely to human psychophysical and perceptual mechanisms, such as auditory masking and ASA, than the IBM. Thirdly, the IRM has some computational advantages, such as facilitating probabilistic frameworks. Fourthly, the equivalent binary mask may always be derived from the ratio mask; the reverse is not possible. Fifthly, studies have shown the IRM to provide small gains over the IBM for BSS (in terms of SNR), but modest 
to large gains for ASR and speech intelligibility. Lastly, hints in the literature that the IRM may lead to a higher audio quality than the IBM by reducing musical noise were confirmed in a brief empirical study. The study showed that although the SNR gain offered by the IRM might be small, and might come at the cost of a slightly reduced interference suppression, a significant gain in OPS can be obtained, together with an improved TPS, APS and SAR. Furthermore, the IRM retains many of its advantages independently of any errors made in estimating source powers.

It is acknowledged that the 'ideal' mask might sometimes be beaten in terms of OPS by a slightly less ideal mask. This might suggest that the goal of CASA should perhaps be an 'almost-ideal' ratio mask. However, current algorithms are likely to produce a small degree of error when used for practical BSS and so if the goal is the IRM then it is likely that an 'almost-ideal' ratio mask is what will be actually produced. Thus, adopting the IRM as the goal of CASA is likely to lead to algorithms delivering optimal OPS.

\section{Acknowledgements}

The authors would like to thank Nicoleta Roman and colleagues for providing the data for Table 4, Nilesh Madhu for providing the data for Table 6 , and Jesper Jensen for providing the data for Table 7

\section{References}

1. Anzalone, M., Calandruccio, L., Doherty, K., Carney, L.: Determination of the potential benefit of time-frequency gain manipulation. Ear and hearing 27(5), 480 (2006)

2. Araki, S., Makino, S., Sawada, H., Mukai, R.: Underdetermined blind separation of convolutive mixtures of speech with directivity pattern based mask and ICA. In: C. Puntonet, A. Prieto (eds.) Independent Component Analysis and Blind Signal Separation, Lecture Notes in Computer Science, vol. 3195, pp. 898-905. Springer, Berlin (2004)

3. Araki, S., Makino, S., Sawada, H., Mukai, R.: Reducing musical noise by a fine-shift overlapadd method applied to source separation using a time-frequency mask. IEEE Int. Conf. Acoust., Speech, Signal Proc. (ICASSP) III, 81-84 (2005)

4. Araki, S., Nesta, F., Vincent, E., Koldovsk, Z., Nolte, G., Ziehe, A., Benichoux, A.: The 2011 signal separation evaluation campaign (SiSEC2011): Audio source separation. In: F. Theis, A. Cichocki, A. Yeredor, M. Zibulevsky (eds.) Latent Variable Analysis and Signal Separation, Lecture Notes in Computer Science, vol. 7191, pp. 414-422. Springer, Berlin/Heidelberg (2012)

5. Araki, S., Sawada, H., Mukai, R., Makino, S.: Blind sparse source separation with spatially smoothed time-frequency masking. In: Int. Workshop Acoust. Echo Noise Control. Paris (2006)

6. Barker, J., Josifovski, L., Cooke, M.P., Green, P.D.: Soft decisions in missing data techniques for robust automatic speech recognition. In: Proc. Int. Conf. Spoken Lang. Proc., pp. 373-376 (2000)

7. Bell, A.J., Sejnowski, T.J.: An information-maximization approach to blind separation and blind deconvolution. Neural computation 7(6), 1129-1159 (1995) 
8. Bregman, A.: The meaning of duplex perception: sounds as transparent objects. In: M.E.H. Schouten (ed.) The Psychophysics of Speech Perception, pp. 95-111. Martinus Nijhoff, Dordrecht (1987)

9. Bregman, A.S.: Auditory Scene Analysis. MIT Press, Cambridge, MA (1990)

10. Brons, I., Houben, R., Dreschler, W.A.: Perceptual effects of noise reduction by timefrequency masking of noisy speech. J. Acoust. Soc. Am. 132(4), 2690-2699 (2012)

11. Brungart, D.S., Chang, P.S., Simpson, B.D., Wang, D.: Isolating the energetic component of speech-on-speech masking with ideal time-frequency segregation. J. Acoust. Soc. Am. 120(6), 4007-4018 (2006)

12. Christensen, H., Barker, J., Ma, N., Green, P.: The chime corpus: a resource and a challenge for computational hearing in multisource environments. In: Proc. Interspeech (2010)

13. Coy, A., Barker, J.: An automatic speech recognition system based on the scene analysis account of auditory perception. Speech Commun. 49(5), 384-401 (2007)

14. Emiya, V., Vincent, E., Harlander, N., Hohmann, V.: Subjective and objective quality assessment of audio source separation. IEEE Trans. Audio, Speech, Lang. Proc. 19(7), 2046-2057 (2011)

15. Ephraim, Y., Malah, D.: Speech enhancement using a minimum-mean square error short-time spectral amplitude estimator. IEEE Trans. Acoust., Speech, Signal Proc. 32(6), 1109-1121 (1984)

16. Ephraim, Y., Malah, D.: Speech enhancement using a minimum mean-square error logspectral amplitude estimator. IEEE Trans. Acoust., Speech, Signal Proc. 33(2), 443-445 (1985)

17. Erkelens, J., Hendriks, R., Heusdens, R., Jensen, J.: Minimum mean-square error estimation of discrete fourier coefficients with generalized gamma priors. IEEE Trans. Audio, Speech, Lang. Proc. 15(6), 1741-1752 (2007)

18. Grais, E., Erdogan, H.: Single channel speech music separation using nonnegative matrix factorization and spectral masks. In: 17th Int. Conf. Digital Signal Proc. (DSP), pp. 1-6 (2011)

19. Hartmann, W., Fosler-Lussier, E.: Investigations into the incorporation of the ideal binary mask in ASR. In: IEEE Int. Conf. Acoust., Speech, Signal Proc. (ICASSP), pp. 4804-4807 (2011)

20. Hendriks, R., Heusdens, R., Jensen, J.: MMSE based noise PSD tracking with low complexity. In: IEEE Int. Conf. Acoust., Speech, Signal Proc. (ICASSP), pp. 4266-4269 (2010)

21. Hu, Y., Loizou, P.C.: Techniques for estimating the ideal binary mask. In: Proc. 11th Int. Workshop Acoust. Echo Noise Control (2008)

22. Jensen, J., Hendriks, R.: Spectral magnitude minimum mean-square error estimation using binary and continuous gain functions. IEEE Trans. Audio, Speech, Lang. Proc. 20(1), 92-102 (2012)

23. Jutten, C., Hérault, J.: Independent component analysis (inca) versus principal component analysis. In: Signal Processing IV: Theories and applications - Proceedings of EUSIPCO, pp. 643-646. North-Holland, Grenoble, France (1988)

24. Lee, D.D., Seung, H.S.: Learning the parts of objects by non-negative matrix factorization. Nature 401(6755), 788-791 (1999)

25. Li, M., McAllister, H., Black, N., De Perez, T.: Perceptual time-frequency subtraction algorithm for noise reduction in hearing aids. IEEE Trans. Biomed. Eng. 48(9), 979-988 (2001)

26. Li, N., Loizou, P.C.: Effect of spectral resolution on the intelligibility of ideal binary masked speech. The Journal of the Acoustical Society of America 123(4), 59-64 (2008)

27. Li, N., Loizou, P.C.: Factors influencing intelligibility of ideal binary-masked speech: Implications for noise reduction. J. Acoust. Soc. Am. 123(3), 1673-1682 (2008)

28. Li, Y., Wang, D.: On the optimality of ideal binary time-frequency masks. Speech Commun. 51(3), 230-239 (2009)

29. Madhu, N., Breithaupt, C., Martin, R.: Temporal smoothing of spectral masks in the cepstral domain for speech separation. IEEE Int. Conf. Acoust., Speech, Signal Proc. (ICASSP) pp. 45-48 (2008) 
30. Madhu, N., Spriet, A., Jansen, S., Koning, R., Wouters, J.: The potential for speech intelligibility improvement using the ideal binary mask and the ideal Wiener filter in single channel noise reduction systems: Application to auditory prostheses. IEEE Trans. Audio, Speech, Lang. Proc. 21(1), 63 -72 (2013)

31. Makkiabadi, B., Sanei, S., Marshall, D.: A k-subspace based tensor factorization approach for under-determined blind identification. In: Forty Fourth Asilomar Conf. Signals, Systems, Comp., pp. 18-22 (2010)

32. Moore, B.C.J.: An Introduction to the Psychology of Hearing, fifth edn. Academic Press, London (2004)

33. Mowlaee, P., Saeidi, R., Martin, R.: Model-driven speech enhancement for multisource reverberant environment (Signal Separation Evaluation Campaign (SiSEC) 2011). In: F. Theis, A. Cichocki, A. Yeredor, M. Zibulevsky (eds.) Latent Variable Analysis and Signal Separation, Lecture Notes in Computer Science, vol. 7191, pp. 454-461. Springer, Berlin/Heidelberg (2012)

34. Naik, G.R., Kumar, D.K.: An overview of independent component analysis and its applications. Informatica 35, 63-81 (2011)

35. Ozerov, A., Vincent, E., Bimbot, F.: A general flexible framework for the handling of prior information in audio source separation. IEEE Trans. Audio, Speech, and Lang. Proc. 20(4), 1118-1133 (2012)

36. Patterson, R., Nimmo-Smith, I., Holdsworth, J., Rice, P.: An efficient auditory filterbank based on the gammatone function. Technical report, MRC Applied Psychology Unit, Cambridge (1987)

37. Pedersen, M., Wang, D., Larsen, J., Kjems, U.: Overcomplete blind source separation by combining ICA and binary time-frequency masking. In: IEEE Workshop Mach. Learn. Signal Proc., pp. 15-20 (2005)

38. Peterson, W., Birdsall, T.G., Fox, W.C.: The theory of signal detectability. In: Proc. of the IRE Prof. Group on Inf. Theory 4, pp. 171-212 (1954)

39. Rangachari, S., Loizou, P.C.: A noise-estimation algorithm for highly non-stationary environments. Speech Commun. 48(2), 220-231 (2006)

40. Roman, N., Wang, D.: Pitch-based monaural segregation of reverberant speech. J. Acoust. Soc. Am. 120(1), 458-469 (2006)

41. Shannon, R., Zeng, F., Kamath, V., Wygonski, J., Ekelid, M.: Speech recognition with primarily temporal cues. Science 270, 303-303 (1995)

42. Srinivasan, S., Roman, N., Wang, D.: Binary and ratio time-frequency masks for robust speech recognition. Speech Commun. 48(11), 1486 - 1501 (2006)

43. Stokes, T., Hummersone, C., Brookes, T.: Reducing binary masking artefacts in blind audio source separation. In: Proc. 134th Audio Eng. Soc. Conv. Rome (2013). Accepted

44. Swets, J.A.: Is there a sensory threshold? Science 134(3473), 168-177 (1961)

45. Swets, J.A.: Signal detection and recognition by human observers. Wiley, New York (1964)

46. Tanner Jr., W.P., Swets, J.A.: A decision-making theory of visual detection. Psychol. Review 61(6), 401-409 (1954)

47. Vincent, E., Gribonval, R., Févotte, C.: Performance measurement in blind audio source separation. IEEE Trans. Audio, Speech, Lang. Proc. 14(4), 1462-1469 (2006)

48. Wang, D.: On ideal binary mask as the computational goal of auditory scene analysis. In: P. Divenyi (ed.) Speech separation by humans and Machines, pp. 181-197. Kluwer Academic, Norwell, MA (2005)

49. Wang, D.: Time-frequency masking for speech separation and its potential for hearing aid design. Trends in Amplification 12(4), 332-353 (2008)

50. Wang, D., Brown, G.J.: Fundamentals of computational auditory scene analysis. In: D. Wang, G.J. Brown (eds.) Computational Auditory Scene Analysis: Principles, Algorithms and Applications, pp. 1-44. John Wiley \& Sons, Hoboken, NJ (2006)

51. Wiener, N.: Extrapolation, Interpolation, and Smoothing of Stationary Time Series: with Engineering Applications. MIT Press (1950) 